International Mathematical Forum, Vol. 8, 2013, no. 13, 627 - 631 HIKARI Ltd, www.m-hikari.com

\title{
Groups with One Nonlinear Irreducible Brauer Character are Solvable
}

\author{
Xiaoyou Chen \\ College of Science \\ Henan University of Technology \\ Zhengzhou 450001, China \\ cxymathematics@hotmail.com \\ Jiwen Zeng \\ School of Mathematical Sciences \\ Xiamen University, Xiamen 361005, China \\ jwzeng@xmu.edu.cn
}

Copyright (c) 2013 Xiaoyou Chen and Jiwen Zeng. This is an open access article distributed under the Creative Commons Attribution License, which permits unrestricted use, distribution, and reproduction in any medium, provided the original work is properly cited.

\begin{abstract}
Let $G$ be a finite group and $p$ be a prime divisor of $|G|$. Denote by $\operatorname{IBr}(G)$ and $\operatorname{LBr}(G)$ the sets of irreducible $p$-Brauer characters and linear $p$-Brauer characters of $G$, respectively. If all the irreducible $p$-Brauer characters of $G$ are linear, that is, $\operatorname{IBr}(G)=\operatorname{LBr}(G)$, then $G$ is solvable. When $G$ has only one nonlinear irreducible $p$-Brauer character, it is proved in this note that $G$ is solvable. If $G$ has two nonlinear irreducible $p$-Brauer characters, however, $G$ is not necessarily solvable.
\end{abstract}

Mathematics Subject Classification: 20C15, 20C20

Keywords: Brauer character, nonlinear irreducible Brauer character, solvable group

\section{Introduction}

Let $G$ be a finite group and $G^{\prime}$ be the derived subgroup of $G$. Denote by $\operatorname{Irr}(G)$ and $\operatorname{Lin}(G)$ the sets of irreducible (complex) characters and linear (complex) 
characters of $G$, respectively. If all the irreducible characters of $G$ are linear, that is, $\operatorname{Irr}(G)=\operatorname{Lin}(G)$, then $G$ is abelian. G. Seitz and J. Pelikán proved that finite groups with only one nonlinear irreducible character are solvable in [7] and [6], respectively. They classified finite groups with only one nonlinear irreducible character from different views. G. Seitz proved that a finite group $G$ with only one nonlinear irreducible character if and only if

(i) $|G|=2^{k}, k$ is odd and $G^{\prime}=\mathbf{Z}(G)$ with order 2 , or

(ii) $G$ is the group of all transformations $v \rightarrow a v+b$ on a finite field $G F(q)$ of order $q>2$, where $a, b \in G F(q)$ and $a \neq 0$.

And J. Pelikán gave a full description of such groups of those two cases in detail. Also, K. Motose [4] considered finite groups with only one nonlinear irreducible character. J. Pelikán and K. Motose both proved that those groups are isomorphic to affine groups over finite fields or central products of dihedral groups $D_{8}$ and quaternion groups $Q_{8}$.

Let $G$ be a finite group and $p$ be a prime divisor of $|G|$. Let $G^{0}$ be the set of $p$-regular elements, that is, $G^{0}=\{g \in G \mid p \nmid o(g)\}$. Denote by $\operatorname{cl}(G)$ and $\operatorname{cl}\left(G^{0}\right)$ the sets of conjugacy classes and $p$-regular classes of $G$, respectively. And we write $\operatorname{IBr}(G)$ and $\operatorname{LBr}(G)$ for the sets of irreducible $p$-Brauer characters and linear $p$-Brauer characters of $G$, respectively. For notational convenience, once a prime number $p$ is chosen we simply write Brauer characters for $p$ Brauer characters and regular classes for $p$-regular classes. For other notations and terminologies, one can refer to [3] and [5].

Inspired by [7] and [6], we consider in this note the case when a finite group $G$ is with the number of nonlinear irreducible Brauer characters not more than 2. In fact, if all the irreducible Brauer characters of $G$ are linear, then $G$ is solvable. Also, we have the following.

Theorem 1.1. Let $G$ be a finite group and $p$ be a prime divisor of $|G|$. If $G$ has exactly one nonlinear irreducible Brauer character, then $G$ is solvable.

If $|\operatorname{IBr}(G)|=|\operatorname{LBr}(G)|+2$, however, we cannot have that $G$ is necessarily solvable. For instance, let $A_{5}$ be the alternating group on five letters and $p=5$; then $A_{5}$ has two nonlinear irreducible Brauer characters. And that is the only one finite simple group which we could find up to date to have the property that it has only two nonlinear irreducible Brauer characters.

\section{Proofs}

It is known by character theory that the number of all linear characters of a finite group $G$ is the index of the derived subgroup $G^{\prime}$ in $G$, that is, $\left|G: G^{\prime}\right|=$ $|\operatorname{Lin}(G)|$. Similarly, for $p$-Brauer characters, we have the following. 
Lemma 2.1. Let $G$ be a finite group and $H$ be the smallest normal subgroup such that $G / H$ is an abelian $p^{\prime}$-group, that is, $H=G^{\prime} \mathbf{O}^{p^{\prime}}(G)$, where $p$ is a prime divisor of $|G|$. Then

(a) ${ }^{0}: \operatorname{Irr}(G / H) \rightarrow \operatorname{LBr}(G), \quad \chi \mapsto \chi^{0}$ is a bijection;

(b) $|\operatorname{LBr}(G)|=\left|G: G^{\prime}\right|_{p^{\prime}}$, where $\left|G: G^{\prime}\right|_{p^{\prime}}$ denotes the $p^{\prime}$-part of $\left|G: G^{\prime}\right|$;

(c) $\operatorname{IBr}(G)=\operatorname{LBr}(G)$ if and only if $P=H$, where $P$ is the Sylow $p$-subgroup of $G$;

(d) $G$ is solvable if $\operatorname{IBr}(G)=\operatorname{LBr}(G)$.

Proof. Let $\lambda \in \operatorname{LBr}(G)$ be afforded by the $F$-representation $\Lambda: G \rightarrow F^{\times}$, where $F$ is the algebraic closure of the prime field $\mathbb{Z}_{p}$ and $F^{\times}=F-\{0\}$. Then $G / \operatorname{ker} \lambda=G / \operatorname{ker} \Lambda \lesssim F^{\times}$. By Lemma 2.1 of [5], it is known that the field $F$ does not contain nontrivial $p$-elements. It follows that $G / \operatorname{ker} \lambda$ is a cyclic $p^{\prime}$-group and then $H \subseteq \operatorname{ker} \lambda$. Thus $\lambda \in \operatorname{IBr}(G / H)$. Since $G / H$ is an abelian $p^{\prime}$-group, we have that $\operatorname{Lin}(G / H)=\operatorname{Irr}(G / H)=\operatorname{IBr}(G / H) \subseteq \operatorname{LBr}(G)$ and hence $\operatorname{IBr}(G / H)=\operatorname{LBr}(G)$. Note that $\chi_{G^{0}}=\chi^{0} \in \operatorname{LBr}(G)$ for every $\chi \in \operatorname{Irr}(G / H)$. And then ${ }^{0}: \operatorname{Irr}(G / H) \rightarrow \operatorname{LBr}(G)$ is a bijection. In fact, since $\operatorname{Irr}(G / H)$ and $\operatorname{LBr}(G)$ are groups, it follows that ${ }^{0}$ is a group isomorphism. Notice that $G / G^{\prime}$ is nilpotent. It follows that

$$
\left|G: G^{\prime}\right|_{p^{\prime}}=\left|G / G^{\prime} / H / G^{\prime}\right|=|G / H|=|\operatorname{LBr}(G)|
$$

and the proofs of (a) and (b) are complete.

If $\operatorname{IBr}(G)=\operatorname{LBr}(G)$, then $G / \operatorname{ker} \varphi$ is a cyclic $p^{\prime}$-group for each $\varphi \in \operatorname{IBr}(G)$. Thus $G^{\prime} \mathbf{O}^{p^{\prime}}(G) \leq \operatorname{ker} \varphi$ and then $G^{\prime} \mathbf{O}^{p^{\prime}}(G) \leq \cap_{\varphi \in \operatorname{IBr}(G)} \operatorname{ker} \varphi=\mathbf{O}_{p}(G)$ by Lemma 2.1 of [1]. Since

$$
P \leq G^{\prime} \mathbf{O}^{p^{\prime}}(G) \leq \mathbf{O}_{p}(G) \leq P,
$$

it follows that $P=H$. Conversely, if $P=H$, then for every $\varphi \in \operatorname{IBr}(G)$ we conclude that $\varphi \in \operatorname{IBr}(G / P)=\operatorname{IBr}(G / H)$. Note that $G / P$ is an abelian $p^{\prime}$-group. Thus $\operatorname{IBr}(G)=\operatorname{LBr}(G)$ by (a) and the proof of (c) is complete.

If $\operatorname{IBr}(G)=\operatorname{LBr}(G)$, it follows that $H=G^{\prime} \mathbf{O}^{p^{\prime}}(G)$ is the Sylow $p$-subgroup of $G$. Also, $G / H$ is an abelian $p^{\prime}$-group and then $G$ is solvable. The proof is complete.

If all the irreducible Brauer characters of the finite group $G$ are linear, we have that $G$ has a Sylow tower by Lemma 2.1. We know that a finite supersolvable group always has a Sylow tower. Is $G$ supersolvable if $\operatorname{IBr}(G)=$ $\operatorname{LBr}(G)$ ? The answer is negative. For example, let $A_{4}$ be the alternating group on four letters and $p=2$; then all the irreducible Brauer characters of $A_{4}$ are linear, but $A_{4}$ is not supersolvable.

Now, we list the following theorem which contains Theorem 1.1 and give the proof. 
Theorem 2.2. Let $G$ be a finite group and $p$ be a prime divisor of $|G|$. Suppose that $G$ have only one nonlinear irreducible Brauer character. Then we have the following.

(i) If $1<K \triangleleft G$ and $K$ is not a p-group, then $G^{\prime} \mathbf{O}^{p^{\prime}}(G) \subseteq P K$, where $P \in \operatorname{Syl}_{p}(G)$.

(ii) $G$ is solvable.

Proof. (i) Let $\varphi$ be the nonlinear irreducible Brauer character of $G$. Notice that $G$ can act by conjugation on $\operatorname{IBr}(K)$, that is, if $\theta \in \operatorname{IBr}(K), g \in G$, then $\theta^{g}(h)=\theta\left(h^{g^{-1}}\right)$. Since $K$ is not a $p$-group, it follows that $G$ has at least two orbits on $\operatorname{IBr}(K)$. Now, by Clifford's theorem, we can take $\theta \in$ $\operatorname{IBr}(K)-\operatorname{IBr}\left(\varphi_{K}\right)$, where $\operatorname{IBr}\left(\varphi_{K}\right)$ denotes the set of all irreducible constituents of $\varphi_{K}$. Again by Clifford's theorem, we conclude that $\varphi$ is not a constituent of $\theta^{G}$, and then all of the irreducible constituents of $\theta^{G}$ are linear. Therefore $\operatorname{IBr}\left(\theta^{G}\right) \subseteq \operatorname{LBr}(G)=\operatorname{IBr}\left(G / G^{\prime} \mathbf{O}^{p^{\prime}}(G)\right)$.

Let $\lambda$ be a linear Brauer constituent of $\theta^{G}$. Then $\lambda_{K}=\theta$. By Gallagher's theorem (see also Corollary 8.20 in [5]), it follows that $\operatorname{IBr}\left(\theta^{G}\right)=\{\lambda \mu \mid \mu \in$ $\operatorname{IBr}(G / K)\}$ and therefore all of the irreducible Brauer characters of $G / K$ are linear. Thus the Sylow $p$-subgroup $P K / K$ of $G / K$ is normal in $G / K$, where $P \in \operatorname{Syl}_{p}(G)$. By Lemma 2.1(c), we have $G / P K$ is an abelian $p^{\prime}$-group, and then $G^{\prime} \mathbf{O}^{p^{\prime}}(G) \subseteq P K$.

(ii) Write $H=G^{\prime} \mathbf{O}^{p^{\prime}}(G)$. We first argue that each nonidentity element of $G / H$ is a disjoint union of regular classes of $G$. Let $a, b \in G^{0}$ be conjugate. Then there exists an element $g \in G$ such that $a^{g}=b$ and thus $a^{-1} g^{-1} a g=$ $a^{-1} b \in G^{\prime} \subseteq H$. It follows that $a, b \in a H$, as wanted.

Now, since $|\operatorname{IBr}(G)|=|\operatorname{LBr}(G)|+1=|G: H|+1$ by the proof of Lemma 2.1 and $|\operatorname{IBr}(G)|=\left|\operatorname{cl}\left(G^{0}\right)\right|$, it follows that $H^{0}$ consists of two regular classes of $G$ which are $\{1\}$ and $H^{0}-\{1\}$. Then the nonidentity regular elements of $H$ have the same prime order. Therefore, $|H|$ is divisible by two distinct primes and by Burnside's $p^{a} q^{b}$ theorem it follows that $H$ is solvable. Thus $G$ is solvable and the proof is complete.

When $\operatorname{IBr}(G)=\operatorname{LBr}(G)$, we have another explanation of $H$ being the Sylow $p$-subgroup of $G$. Note that $|\operatorname{IBr}(G)|=|G: H|$ and similar to the above proof it follows that $H$ contains only one regular class $\{1\}$ of $G$. Thus $H$ is a $p$-group and then $H$ is the Sylow $p$-subgroup of $G$.

Note We do not require the condition that $\mathbf{O}_{p}(G)=1$ to prove the solvability of $G$. Notice that Dolfi and Navarro [2] prove that the groups with one nonlinear irreducible Brauer character are solvable under the condition that $\mathrm{O}_{p}(G)=1$. In general, if $G$ has only one nonlinear irreducible Brauer character, we do not necessarily have that $\mathbf{O}_{p}(G)=1$. For example, let $G=C_{3} \rtimes C_{4}$ with order 12 and $p=2$; then $|\operatorname{IBr}(G)|=|\operatorname{LBr}(G)|+1$, but $\mathbf{O}_{2}(G) \cong C_{2}$. 
Acknowledgements. The first author thanks the support of the Doctor Foundation of Henan University of Technology (Grant No. 2010BS048) and Tianyuan Foundations $(11226046,11226158)$. And the second author thanks the support of the National Natural Science Foundation (11261060) and the Fujian Natural Science Foundation (2012J01022).

\section{References}

[1] X. Y. Chen and J. W. Zeng, Super-Brauer characters and super-regular classes, Monatsh. Math., 163 (2011), 15-23.

[2] S. Dolfi and G. Navarro, Finite groups with only one non-linear irreducible representation, Comm. Algebra, to appear.

[3] I. M. Isaacs, Character Theory of Finite Groups, Academic Press, New York (1976).

[4] K. Motose, Finite groups having exactly one non-linear irreducible character, Bull. Fac. Sci. Tech. Hirosaki Univ., 9 (2007), 101-113.

[5] G. Navarro, Characters and Blocks of Finite Groups, Cambridge University Press, Cambridge (1998).

[6] J. Pelikán, Finite groups with few non-linear irreducible characters, Acta. Math. Acad. Sci. Hungar., 25 (1974), 223-226.

[7] G. Seitz, Finite groups having only one irreducible representation of degree greater than one, Proc. Amer. Math. Soc., 19 (1968), 459-461.

\section{Received: January 11, 2013}

